\title{
Interacción térmica recubrimiento- substrato en la proyección a alta velocidad (HVOF) de partículas (polvo) de WC-Co ${ }^{(\bullet)}$
}

\author{
V.V. Sobolev ${ }^{(*)}$, J.M. Guilemany ${ }^{(*)}$ y J.A. Calero ${ }^{(*)}$
}

\begin{abstract}
Resumen Se utiliza la simulación matemática para establecer la interacción térmica entre un substrato y un recubrimiento obtenido mediante proyección térmica de alta velocidad, HVOF. El substrato es un acero 34CrMo4(UNS-G41350) y el recubrimiento está formado por la solidificación de gotas semifundidas de partículas de polvo de WC-12\% Co. El análisis del proceso de transferencia de calor permite la investigación de la evolución de la temperatura, la solidificación del recubrimiento, la fusión y posterior solidificación del substrato, las características peculiares de la interacción térmica entre el substrato y la primera capa de recubrimiento, así como con las diferentes capas sucesivas, y la estimación de las condiciones óptimas para la formación de la estructura del substrato y del recubrimiento. Los resultados obtenidos se han utilizado en posteriores artículos para predecir parámetros estructurales que están, por su parte, en concordancia con los datos experimentales.
\end{abstract}

Palabras clave: Proyección HVOF. Simulación matemática. Transferencia de calor. Solidificación. Condiciones óptimas.

\section{Thermal interaction coating-substrate in high velocity oxyfuel (HVOF) spraying of WC-Co powder particles}

\begin{abstract}
The mathematical simulation of the thermal interaction between a 34CrMo4 (UNS-G41350) steel substrate and a coating formed by the droplets of WC- $12 \%$ Co powder particles during HVOF spraying is undertaken. Analysis of the heat transfer processes permitted the investigation of the temperature evolution, coating solidification, substrate fusion and solidification, particular features of the thermal interactions between the substrate and the coating as well as between the sucessive coating layers. The analysis has also permitted to estimate the optimal conditions of the substrate and the coating structure formation. The obtained results were used in subsequent articles to predict the structure parameters, which agree with the experimental data.
\end{abstract}

Keywords: HVOF spraying. Mathematical simulation. Heat transfer. Solidification. Optimal conditions.

\section{INTRODUCCIÓN}

La proyección térmica de alta velocidad, HVOF, es una de las más importantes técnicas en el desarrollo de recubrimientos protectores $(1$ y 2$)$. Su eficiencia depende en gran medida de la interacción térmica substrato-recubrimiento, que determina su estructura y las propiedades de la región interfacial e influye esencialmente en la cinética de la construcción del recubrimiento.

(•) Trabajo recibido el día 20 de septiembre de 1994.

${ }^{*}$ Metalurgia Física-Ciencia de Materiales. Dpto. de Ingeniería Química y Metalurgia. Universidad de Barcelona. Martí i Franqués, 1. 08028-Barcelona (España).
La simulación matemática del proceso de proyección HVOF ofrece amplias posibilidades de mejorar la citada tecnología, teniendo en cuenta que la medición de los parámetros de interacción térmica es difícil y cara.

El objetivo de este artículo es investigar la interacción térmica substrato-recubrimiento mediante un modelo matemático que refleje las principales características del proceso. Los resultados obtenidos, juntos con los del modelo para el comportamiento térmico y el movimiento de la partícula, así como los obtenidos para la formación de la porosidad en el recubrimiento (3-7), contribuyen al establecimiento de condiciones óptimas para el proceso de proyección HVOF. 


\section{DESCRIPCIÓN DEL MODELO}

Durante los últimos años, se han desarrollado diversos modelos matemáticos para describir la formación del recubrimiento en el proceso de proyección por plasma (8 y 9). Debido a las relativamente bajas velocidades, la formación del recubrimiento puede considerarse en este caso, como resultado de sucesivos choques de gotas en la superficie del substrato (10).

Durante la proyección HVOF, las velocidades de las partículas son significativamente mayores y, por tanto, el movimiento colectivo de partículas y el choque de estas adquieren una mayor importancia. Como resultado, la densidad del recubrimiento crece y la progresiva deposición de las gotas forma capas más delgadas en comparación con las obtenidas por proyección plasma (1).

En este trabajo se considera la proyección HVOF de dos partículas de polvo diferentes, constituidas por WC en una matriz metálica de Co-40\% W y WC en una fase metálica pura (Co), que se proyectan sobre la superficie de un cilindro de acero $34 \mathrm{CrMo}$. Esta proyección se ha efectuado utilizando un equipo de HVOF (Plasma Technik DT 100) instalado en la Universidad de Barcelona en el que una plataforma con los mencionados cilindros gira con un intervalo de tiempo de $\theta=$ $0,16 \mathrm{~s}$ entre la aplicación de dos capas sucesivas (Fig. 1).

\subsection{Transferencia de calor}

Debido a que la velocidad de las partículas durante la proyección HVOF es muy elevada, el grosor de la capa de recubrimiento es considerablemente menor que sus dimensiones longitudinales (1

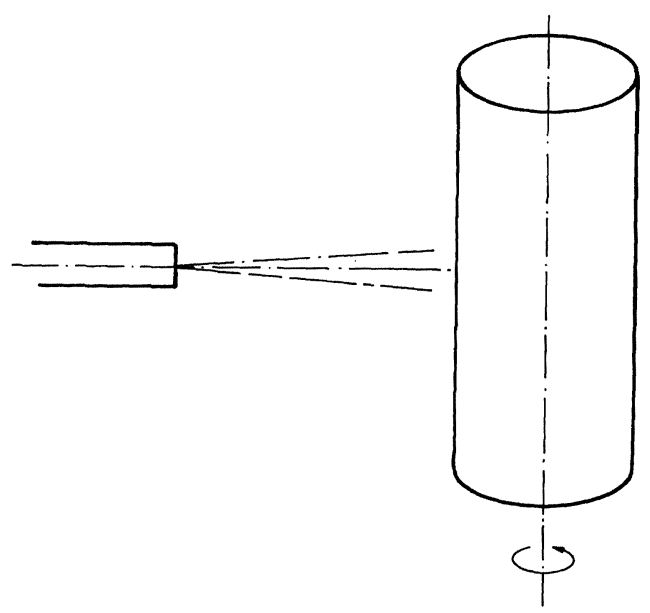

FIG. 1.- Representación esquemática de la proyección HVOF.

Fig. 1.- Scheme of HVOF spraying. y 2). Se puede considerar, por lo tanto, que el campo de temperaturas en el substrato $T_{1}$ y en el recubrimiento $T_{2}$ es función del tiempo $t$ y de la coordenada transversal $r$ (Fig. 2). En particular esto es válido en la región central de la superficie proyectada.

El problema de la transferencia de calor se considera a través del sistema cilíndrico de coordenadas con simetría acimutal y el origen se establece en el centro de rotación del cilindro.

En la superficie exterior de la capa de recubrimiento $\left(r=R_{1}\right)$ se aplica la condición límite que describe el intercambio de calor gas-gota. Puesto que el grosor del substrato $\delta_{1}$ es mucho mayor que el de la capa de recubrimiento, $\delta_{2}=R_{1}-R$, es evidente que a una cierta distancia, $r=R_{*}$, del centro del substrato su temperatura permanecerá inamovible bajo la acción del intercambio de calor substrato-recubrimiento. Esto significa que podemos considerar la temperatura del substrato, $T_{1}$, igual a su valor inicial $T_{10}$.

La solidificación de la capa de recubrimiento así como la fusión de la parte superior del substrato y su posterior solidificación se han tenido en cuenta mediante la formulación del problema de Stefan en el caso de un metal puro y con la introducción de un calor específico efectivo de acuerdo con (11) cuando se considera que cambia la fase en la aleación.

La resistencia térmica en la interfase substratorecubrimiento es aproximadamente de $10^{-4}-10^{6}$ $\mathrm{W}^{-1} \mathrm{~m}^{2} \mathrm{~K}$ y su valor exacto es difícil de medir o calcular puesto que depende de innumerables factores

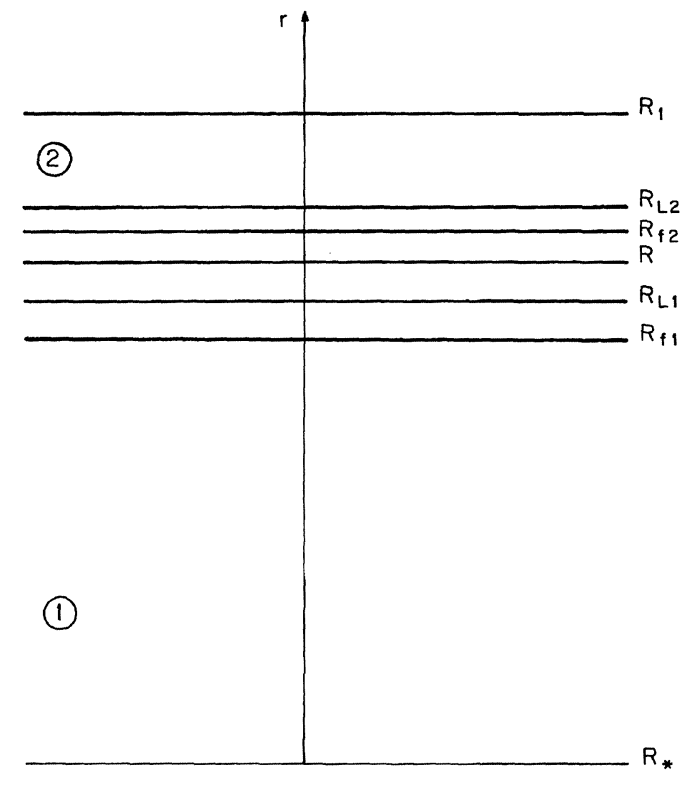

FIG. 2.- Sistema de coordenadas, límites y planos isotérmicos (superficies).

Fig. 2.- Coordinate system, boundary and isothermal planes (surfaces). 
tales como, rugosidad de la superficie, presencia de óxidos en la superficie, etc. Debido a esto, la simulación matemática se utiliza frecuentemente para ajustar parámetros (9 y 10).

Esta resistencia térmica se manifiesta en una acusada disminución de la transferencia de calor desde el recubrimiento a la interfase. Asimismo, es posible tenerla en cuenta mediante la introducción de una conductividad de calor efectiva $\gamma \lambda_{2}$ a la distancia $L=r+L_{2}\left(L_{2}<R\right)$. Asumiendo lo citado anteriormente se pueden utilizar las condiciones de frontera de cuarto orden correspondientes a un contacto térmico ideal en la interfase substrato-recubrimiento.

Debido a esto, la formulación del problema de tranferencia de calor en condiciones de contacto térmico ideal en la interfase substrato-recubrimiento $(r=R)$ incluye dos ecuaciones de conductividad de calor para $T_{1}, T_{2}$ y la correspondiente condición frontera y condiciones iniciales:

$$
\begin{aligned}
& \rho_{1} c_{1} \psi_{1} \frac{\partial T_{1}}{\partial t}=\frac{1}{r} \frac{\partial}{\partial r}\left(r \lambda_{1} \frac{\partial T_{1}}{\partial r}\right), R_{*} \leq r \leq R \\
& \rho_{2} c_{2} \psi_{2} \frac{\partial T_{2}}{\partial t}=\frac{1}{r} \frac{\partial}{\partial r}\left(r \lambda_{2} \frac{\partial T_{2}}{\partial r}\right), R \leq r \leq R_{1}
\end{aligned}
$$

$\psi_{1}=1+q_{\mathrm{i}} c_{\mathrm{i}}^{1}\left(1-\kappa_{\mathrm{i}}\right)^{-1}\left(T_{\mathrm{ci}}-T_{\mathrm{i}}\right)^{-1}\left(\frac{T_{\mathrm{ci}}-T_{\mathrm{Li}}}{T_{\mathrm{ci}}-T_{\mathrm{i}}}\right)^{\frac{2-\kappa_{\mathrm{i}}}{1-\kappa_{\mathrm{i}}}}$

$$
T_{\mathrm{fi}} \leq T_{\mathrm{i}} \leq T_{\mathrm{Li}} ; \quad \psi_{\mathrm{i}}=1, \quad T_{\mathrm{i}}>T_{\mathrm{Li}}, \quad T_{\mathrm{i}}<T_{\mathrm{fi}} ; \quad i=1,2
$$

$$
\begin{gathered}
T_{1}\left(R_{*}, t\right)=T_{10} \\
T_{1}(R, t)=T_{2}(R, t), \quad \frac{\partial T_{1}}{\partial r}(R, t)=\frac{\partial T_{2}}{\partial r}(R, t) \\
\lambda_{2} \frac{\partial T_{2}}{\partial r}\left(R_{1}, t\right)=-\alpha\left[T_{2}\left(R_{1}, t\right)-T_{\mathrm{b}}\right] \\
T_{1}(r, 0)=T_{10} \\
T_{2}(r, t)=T_{20}
\end{gathered}
$$

El problema de Stefan, en el caso de metal puro, se resuelve teniendo en cuenta un balance de calor en la interfase móvil líquido-sólido $r=R_{\mathrm{c}}(11)$ :

$\lambda_{\mathrm{si}}\left(\frac{\partial T_{\mathrm{si}}}{\partial r}\right)_{\mathrm{r}=\mathrm{R}_{\mathrm{fi}}}-\lambda_{\mathrm{li}}\left(\frac{\partial T_{\mathrm{li}}}{\partial r}\right)_{\mathrm{r}=\mathrm{R}_{\mathrm{fi}}}=q \rho_{\mathrm{si}} \frac{\mathrm{d} R_{\mathrm{ci}}}{\mathrm{d} t}, i=1,2[8]$
Existe otra condición frontera que se aplica en el caso de la interfase líquido-sólido, según:

$$
T_{\mathrm{si}}\left(R_{\mathrm{ci}}, t\right)=T_{\mathrm{li}}\left(R_{\mathrm{ci}}, t\right)=T_{c}, i=1,2
$$

La solución del problema térmico [1]-[9] describe la interacción térmica substrato-recubrimiento que se asocia con la primera capa. Se considera que la segunda capa aparece en el momento correspondiente al período de la rotación del substrato $\theta$.

Por lo tanto, el problema [1]-[9] se debe resolver en el intervalo de tiempo $0 \leq \mathrm{t}<\theta$. Cuando $t=\theta$, se añade una ecuación de conducción de calor similar a [2] en el intervalo de espacio $R_{1}<r \leq R_{1}+\delta$ con condiciones de contacto térmico ideal en la interfase $r=$ $R_{1}$ entre la primera y la segunda capa de recubrimiento. En este caso, la condición frontera [5] se aplica a la superficie externa de la segunda capa $\left(r=R_{1}+\delta\right)$. Este procedimiento, si se repite para todas las capas sucesivas, permite describir aproximadamente el proceso de la construcción del recubrimiento.

\subsection{Criterios de estado térmico}

A partir de los fundamentos de los campos térmicos, se han determinado los criterios $I_{1}$ e $I_{2}$ del estado térmico de la solidificación del metal. Dichos criterios desempeñan un importante papel en la calidad del substrato y de la primera capa de recubrimiento (11 y 12):

$$
\begin{aligned}
& I_{11}=\left(t_{\mathrm{f} 1}-t_{\mathrm{f} 10}\right)^{-1} \int_{t_{\mathrm{f} 10}}^{t_{\mathrm{f} 1}} \frac{\mathrm{d} t}{R^{2}-R_{\mathrm{f} 1}^{2}} \int_{R_{\mathrm{f} 1}}^{R}\left|\frac{\partial T}{\partial r}\right| r \mathrm{~d} r \\
& I_{12}=\left(t_{\mathrm{f} 2}-t_{\mathrm{f} 20}\right)^{-1} \int_{t_{\mathrm{f} 20}}^{t_{\mathrm{f} 2}} \frac{\mathrm{d} t}{R_{1}^{2}-R_{\mathrm{f} 2}^{2}} \int_{R_{\mathrm{f} 2}}^{R_{1}}\left|\frac{\partial T}{\partial r}\right| r \mathrm{~d} r
\end{aligned}
$$

$I_{21}=\left(t_{\mathrm{L} 1}-t_{\mathrm{f} 1}\right)^{-1} \int_{t_{\mathrm{f} 1}}^{t_{\mathrm{L} 1}} \frac{\mathrm{d} t}{R_{\mathrm{L} 1}^{2}-R_{\mathrm{f} 1}^{2}} \int_{R_{\mathrm{f} 1}}^{R_{\mathrm{L} 1}} \tau r \mathrm{~d} r$

$I_{22}=\left(t_{\mathrm{f} 2}-t_{\mathrm{L} 2}\right)^{-1} \int_{t_{\mathrm{L} 2}}^{t_{\mathrm{f} 2}} \frac{\mathrm{d} t}{R_{\mathrm{f} 2}^{2}-R_{\mathrm{L} 2}^{2}} \int_{R_{\mathrm{L} 2}}^{R_{\mathrm{f} 2}} \tau r \mathrm{~d} r$

Los valores de $I_{11}$ e $I_{12}$ definen los gradientes de temperatura integrados medios, que aumentan durante la solidificación en la fase sólida del substrato y en la primera capa de recubrimiento, respectivamente, y son responsables de la formación de tensiones térmicas. Los criterios $I_{21}$ e $I_{22}$ determinan el valor del tiempo medio de residencia de la substancia en la zona de dos fases, que determina a su vez la posibilidad de formación de defectos en la estructura (porosidad, inclusiones no metálicas, etc.) 


\section{SIMULACIÓN MATEMÁTICA}

El problema térmico [1]-[9] se resolvió numéricamente mediante el método de diferencias finitas en la forma implícita con absoluta estabilidad. El algoritmo numérico es similar al descrito en (11).

Se han estudiado los resultados de la proyección térmica de las partículas de polvo WC-(Co-40\% W) y WC-Co . Estas partículas son agregados constituidos por pequeños núcleos de carburos rodeados por metal enlazante. El tamaño de los carburos $(2-3 \mu \mathrm{m})$ permite considerar de manera casi homogénea cada capa de recubrimiento puesto que tienen un grosor de $15 \mu \mathrm{m}$ en las condiciones de proyección existentes. Para investigar la formación de las capas de recubrimiento se deben considerar las propiedades térmicas del conjunto de la mezcla líquido-sólida (3 y 13). Las propiedades del acero se han tomado de (14).

En la versión básica de los cálculos se han introducido los siguientes parámetros:

$\delta_{1}=R-R_{*}=3 \mathrm{~mm}, \quad T_{10}=20^{\circ} \mathrm{C}, \quad T_{\mathrm{b}}=500^{\circ} \mathrm{C}$,

$\delta_{2}=R_{1}-R=15 \mu \mathrm{m}, T_{20}=1.485^{\circ} \mathrm{C}, \gamma=0,0001$, $\delta=\delta_{2}, \quad \alpha=1.000 \mathrm{Wm}^{-2} \mathrm{~K}^{-1}, \quad L_{2}=0,6 \mu \mathrm{m}$.

\section{RESULTADOS Y DISCUSIÓN}

\subsection{Primera capa de recubrimiento}

El líquido inicial está sujeto a un posterior enfriamiento y solidificación. El enfriamiento principal viene dado por la capacidad de acumulación de calor del substrato, asimismo se produce otro tipo de enfriamiento mediante el intercambio de calor entre la superficie de la capa exterior del recubrimiento y el fluido que lo rodea. Debido a la competición entre ambos tipos de enfriamiento, los perfiles de temperatura en la primera capa de recubrimiento alcanzan un valor máximo $T_{\mathrm{m}}$ desplazado hacia la interfase substrato-capa de recubrimiento. El tiempo característico es $t_{\mathrm{c}}=0,96 \mathrm{~s}$.

Este valor máximo se hace menor con el tiempo y con la transición desde la solidificación de la aleación a la de cobalto puro. La posición $r_{\mathrm{m}}$ de $T_{\mathrm{m}}$ se mueve en la dirección de la intercara substrato-recubrimiento (Fig. 3). La velocidad de este movimiento es mayor en el caso de la aleación Co-40 \% W.

El tiempo de solidificación de la capa de recubrimiento $t_{\mathrm{s} 2}$ crece con respecto a las temperaturas iniciales, tanto del substrato como de la capa de recubrimiento. Este tiempo aumenta cuando se incrementa el grosor de la capa y disminuye $\alpha$ (Fig.4). La forma de crecimiento de $t_{\mathrm{s} 2}$ en el intervalo $\delta=15-18 \mu \mathrm{m}$ con la formación de una pequeña zona de dos fases extrayendo el calor latente en el proceso de solidificación, ocurre debido a la ace- leración del movimiento de la isoterma del final de la solidificación $T_{\mathrm{f} 2}$ en la capa de recubrimiento.

Del comportamiento del gradiente térmico integral medio, $I_{12}$, en la fase sólida del recubrimiento (Fig. 5) se puede ver que $I_{12}$ crece con el aumento del grosor $\delta$ de la capa de recubrimiento y conduce a la suavización de la variación de temperatura de la capa en el proceso de la extracción del calor mediante la acumulación de calor por parte del substrato. El incremento de $I_{12}$ con respecto a $\delta$ tiene lugar en la misma región (Fig. 4) y sus causas residen en la misma razón que en el caso de $t_{\mathrm{s} 2}$.

La disminución de $I_{12}$ con $\alpha$ se produce debido al hecho de que el aumento de $\alpha$ conduce a la aceleración del desplazamiento del valor máximo de la temperatura $T_{\mathrm{m}}$ hacia el substrato y, por lo tanto, la suavización de la distribución de la temperatura en el recubrimiento. La forma de la disminución de $I_{12}$ en el intervalo $\alpha=800-850 \mathrm{Wm}^{-2} \mathrm{~K}^{-1}$ se debe a que la región de dos fases crece causando una mayor uniformidad en la distribución de las temperaturas de la capa de recubrimiento como resultado de la extracción de calor latente.

Del comportamiento de $I_{12}$ con respecto a $T_{10} \mathrm{y}$ $T_{20}$ se puede deducir que el valor de $I_{12}$ disminuye con $T_{10}$ y varía de forma no uniforme con respecto a $T_{20}$ alcanzando su valor máximo a la temperatura $T_{20}=1.480{ }^{\circ} \mathrm{C}$ donde tiene lugar la máxima extracción de calor latente en el substrato.

Como ya se ha mencionado con anterioridad, los valores mínimos del gradiente medio de temperaturas $I_{12}$ corresponden a la formación de tensiones térmicas mínimas en la primera capa de recubrimiento durante el proceso de proyección térmica. Desde este punto de vista, se puede predecir que los mejores resultados, se producen, aproximadamente, con los siguientes parámetros del proceso: $\alpha=850-1.000 \mathrm{Wm}^{-2} \mathrm{~K}^{-1} ; \delta=5-15 \mu \mathrm{m} ; T_{10}=600$ ${ }^{\circ} \mathrm{C}$ y $T_{20}=1.475^{\circ} \mathrm{C}$ y $1.500^{\circ} \mathrm{C}$.

La figura 6 muestra que el valor de la integral media de tiempos de residencia en zona de dos fases, $I_{22}$, en un primer momento disminuye con $\delta$, alcanza su valor mínimo y, posteriormente, aumenta, decreciendo después de alcanzar la posición máxima. El criterio $I_{22}$ exhibe un comportamiento no uniforme al aumentar $\alpha$ al igual que con respecto a $T_{10}$ y $T_{20}$.

Teniendo en cuenta que la estructura óptima del recubrimiento obtenida durante la solidificación corresponde al valor mínimo de $I_{22}$, es posible predecir que esta estructura se puede obtener cuando $\delta=9-11 \mu \mathrm{m}, \alpha=850-1.000 \mathrm{Wm}^{-2} \mathrm{~K}^{-1}, T_{10}=100$ ${ }^{\circ} \mathrm{C}, 550{ }^{\circ} \mathrm{C} ; T_{20}=1.483^{\circ} \mathrm{C}$.

Para obtener los valores mínimos de los criterios $I_{12}$ e $I_{22}$ en el mismo proceso se deben combinar las condiciones óptimas para ambos casos (12). Debido a ello, en el desarrollo del control de la solidificación constituye un problema la selección de las 


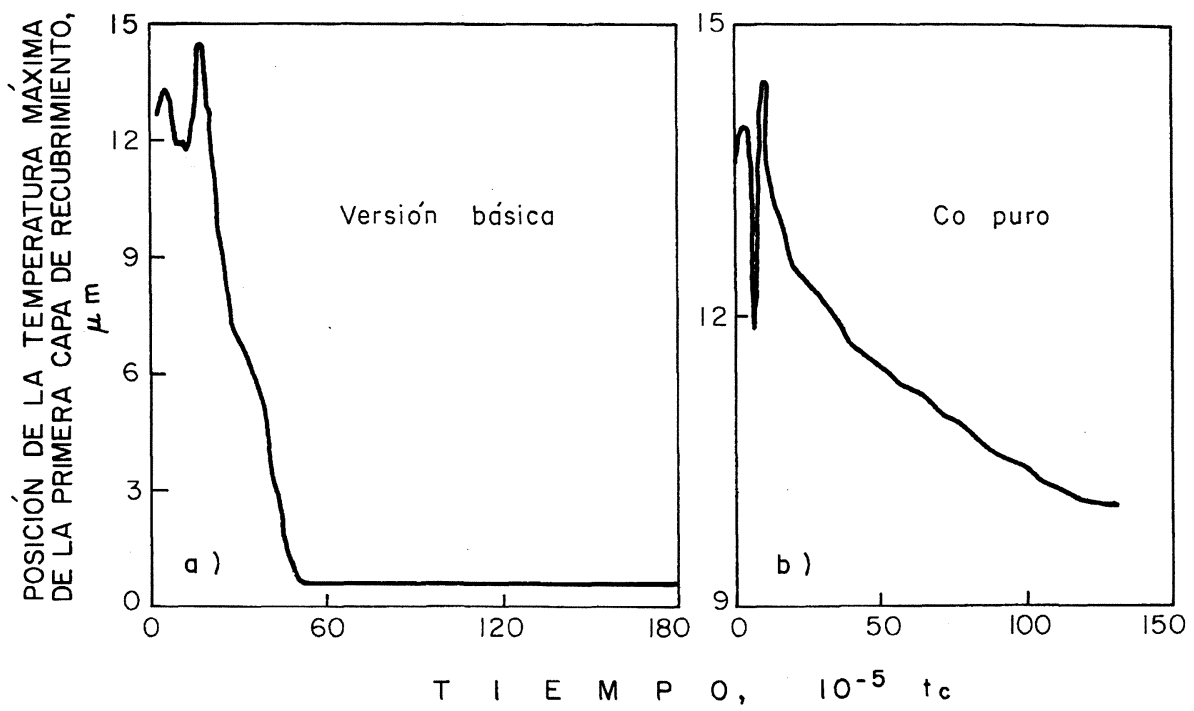

FIG. 3.- Perfiles de temperatura de la primera capa de recubrimiento en diferentes momentos de tiempo para la variante básica (a) y cobalto puro (b).

Fig. 3.- Temperature profiles of the coating first layer at different moments of time for basic version (a) and pure cobalt $(b)$.

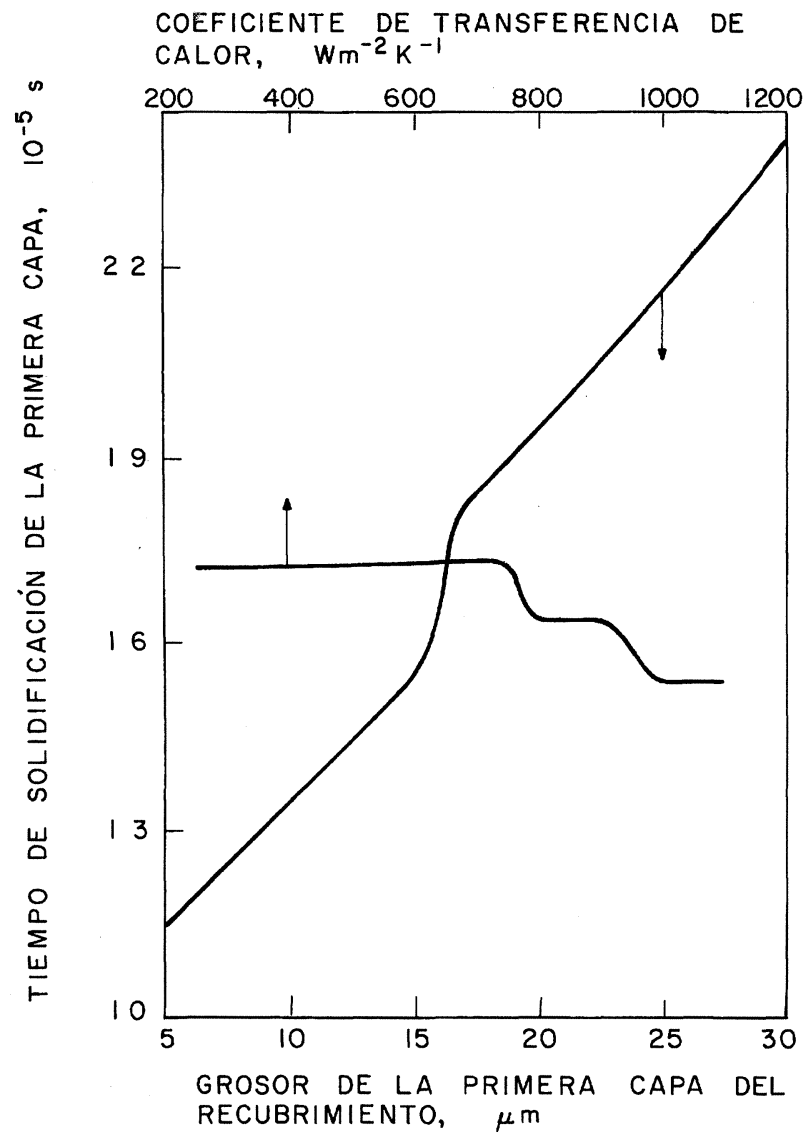

FIG. 4.- Variación del tiempo de solidificación de la primera capa con respecto a su grosor y a $\alpha$.

Fig. 4.- Variation of the first layer solidification time with respect to its thickness and $\alpha$.

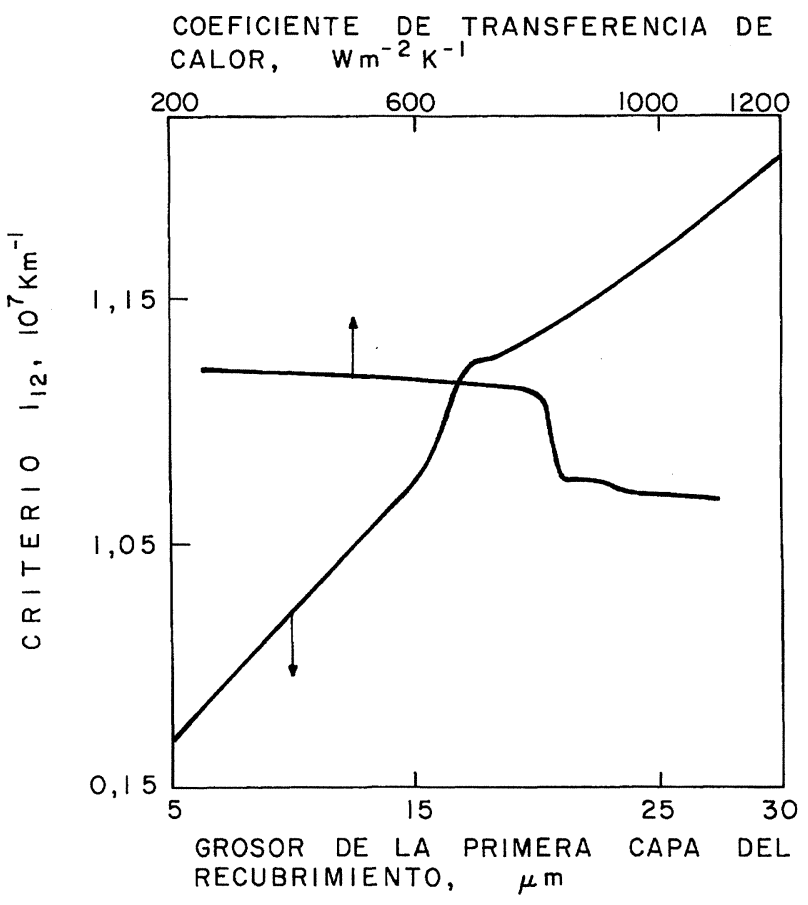

FIG. 5.- Variación del gradiente de temperatura integral medio en la fase sólida de la primera capa con respecto a su grosor y a $\alpha$.

Fig. 5.- Variation of mean integral value of thermal gradient in the first layer solidified solid phase with respect to its thickness and $\alpha$.

condiciones bajo las cuales se puedan obtener los valores más próximos a los mínimos para los criterios $I_{12}$ e $I_{22}$. En el presente caso, dichas 


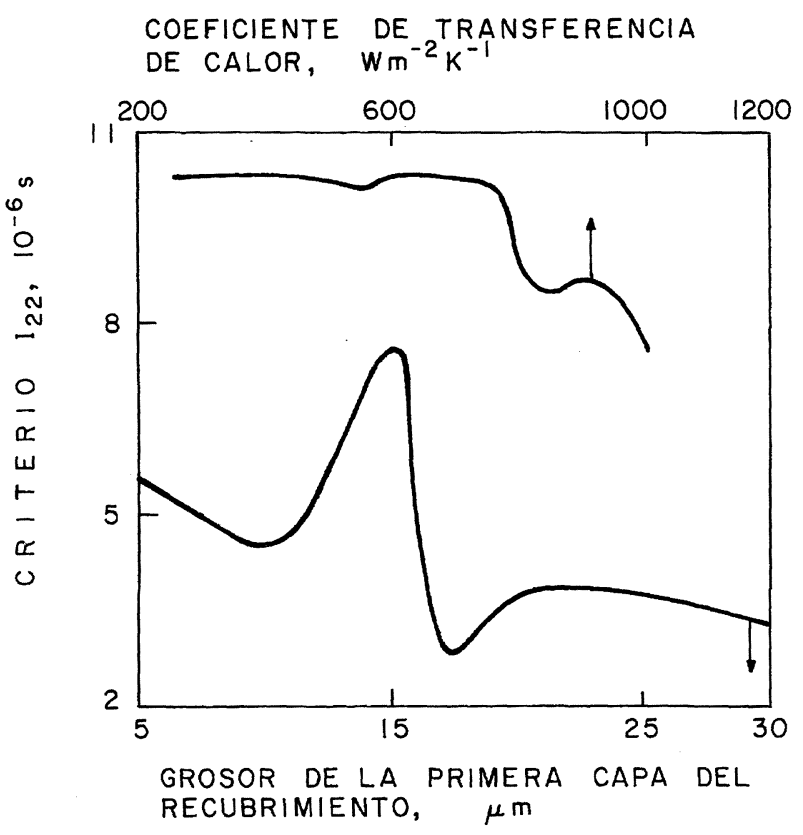

FIG. 6.- Variación del tiempo de residencia integral medio en la zona de dos fases para la primera capa con respecto al grosor y a $\alpha$.

Fig. 6.- Variation of mean integral value of solidifying metal residence time in mushy zone of the first layer with respect to its thickness and $\alpha$.

condiciones podrían corresponder a: $\alpha=850$ $1.000 \mathrm{Wm}^{-2} \mathrm{~K}^{-1} ; T_{10}=20,150$ y $300{ }^{\circ} \mathrm{C} ; T_{20}=$ $1.483-1.485{ }^{\circ} \mathrm{C} ; \delta=9-11 \mu \mathrm{m}$.

En cada caso particular es recomendable estimar la importancia de los diferentes defectos estructurales en el recubrimiento para elegir el que se presente en mayor número en cada caso, por ejemplo, tensiones residuales y ajustar los parámetros de proyección para minimizarlas. En este caso corresponde a la obtención de valores mínimos de $I_{12}$.

\subsection{Substrato}

La historia térmica del substrato, en general, incluye su calentamiento debido a la transferencia de calor desde la primera capa de recubrimiento, su fusión y su posterior solidificación. La zona de dos fases se caracteriza por el movimiento de la isoterma $T=T_{\mathrm{f} 1}$ que puede describirse con respecto al tiempo mediante el parámetro $h=R-R_{\mathrm{f}}$, determinando su valor máximo, $h_{\mathrm{m}}$, la profundidad de la zona de dos fases en el substrato.

El valor $h$ crece con el tiempo, alcanzando su máximo, $h_{\mathrm{m}}$, y disminuyendo después (Fig. 7). El aumento de la curvatura de la superficie del substrato provoca un incremento del calor transferido como resultado de la focalización de los flujos térmicos y, por lo tanto, el aumento de $h_{\mathrm{m}}$ y del tiempo, $t_{\mathrm{m}}$, en el cual se alcanza dicho máximo. Los valores

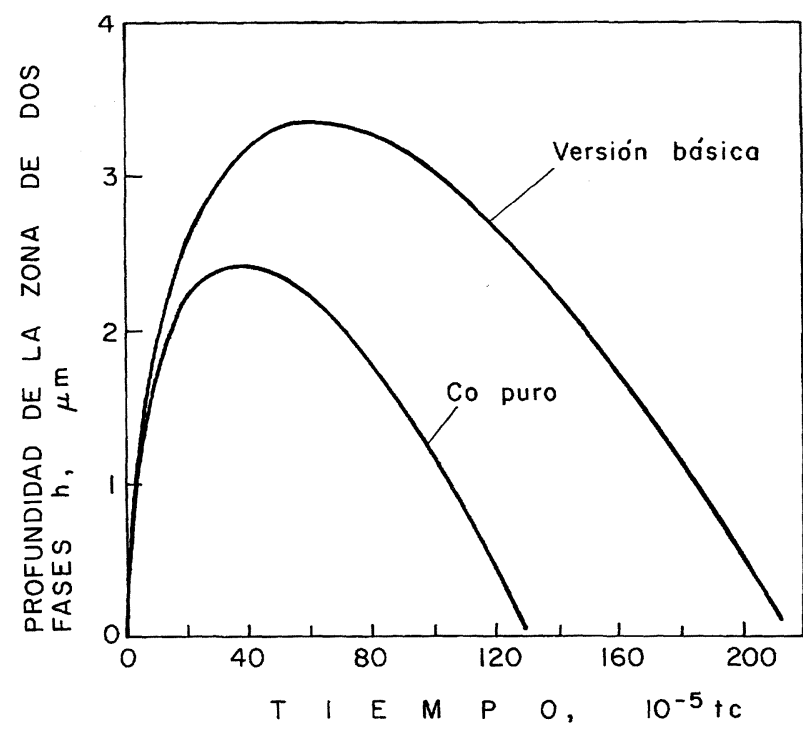

FIG. 7.- Evolución del proceso de fusión del substrato.

Fig. 7.-Time evolution of substrate fusion process.

de $h_{\mathrm{m}}$ y de $t_{\mathrm{m}}$ disminuyen en el caso de cobalto puro, puesto que la temperatura de fusión es superior a la de la aleación Co-40 \% W.

$\mathrm{El}$ incremento de la temperatura inicial del substrato y de la capa de recubrimiento provoca un aumento de $h_{\mathrm{m}}$ (Fig. 8). El comportamiento no uniforme de $h_{\mathrm{m}}$ en las proximidades de $1.480{ }^{\circ} \mathrm{C}$,

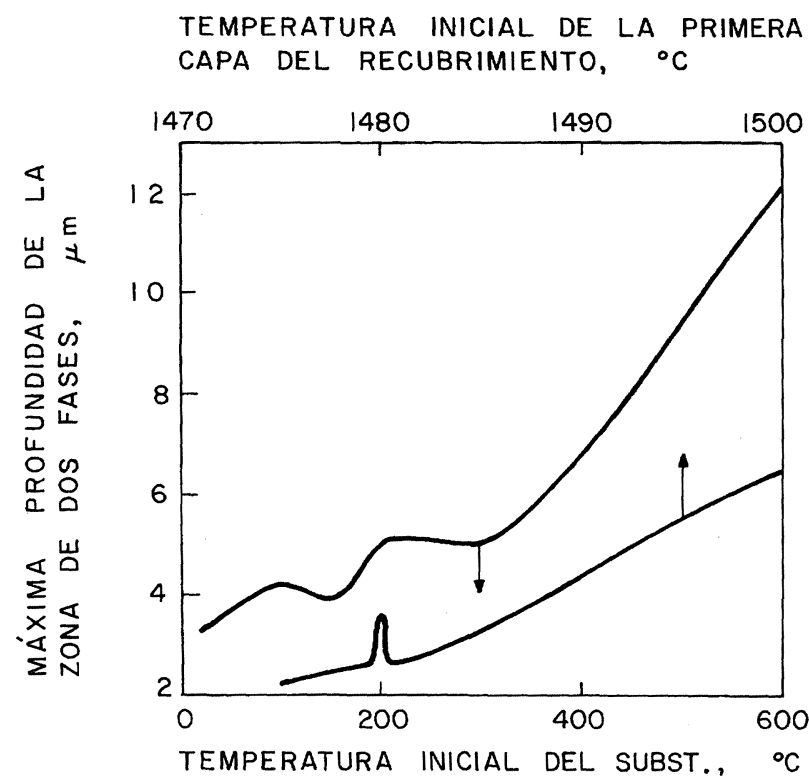

Fig. 8. - Variación de la máxima profundidad de la zona de dos fases en el substrato con respecto a la temperatura inicial del substrato y de la primera capa.

Fig. 8.-Variation of substrate mushy zone maximal depth with respect to the first layer and substrate initial temperatures. 
siendo ésta la temperatura de la isoterma de líquidus del substrato $T_{\mathrm{L} 1}$, se explica por la fusión inmediata del substrato cuando $T_{20}=T_{\mathrm{L} 1}$. El valor de $h_{\mathrm{m}}$ también crece con $T_{10}$. El aumento de $h_{\mathrm{m}}$ con el incremento del grosor de la capa de recubrimiento, $\delta$, y la disminución de $\alpha$, es, evidentemente, resultado del crecimiento de la energía térmica de la capa de recubrimiento (Fig. 9). Desde las figuras 10 y 11 se puede observar que el tiempo de solidificación del substrato, $t_{\mathrm{s} 1}$, se comporta con respecto a $\delta$, $\alpha, T_{10}$ y $T_{20}$ de la misma forma que lo hace $t_{\mathrm{s} 2}$.

El gradiente térmico integral medio, $I_{11}$, para el substrato muestra un comportamiento no uniforme con respecto al grosor de capa, $\delta$ (Fig. 12). Después de un suave crecimiento $I_{11}$ disminuye debido al acusado aumento de la fase sólida de la capa de recubrimiento y aumenta con la distancia entre la isoterma sólidus del substrato y la zona de dos fases de la capa de recubrimiento, donde la extracción de calor latente tiene lugar bajo la condición de un crecimiento muy pequeño de la fase sólida del substrato. Esto ocurre en el intervalo de $5 \mu \mathrm{m} \leq \delta \leq 10 \mu \mathrm{m}$.

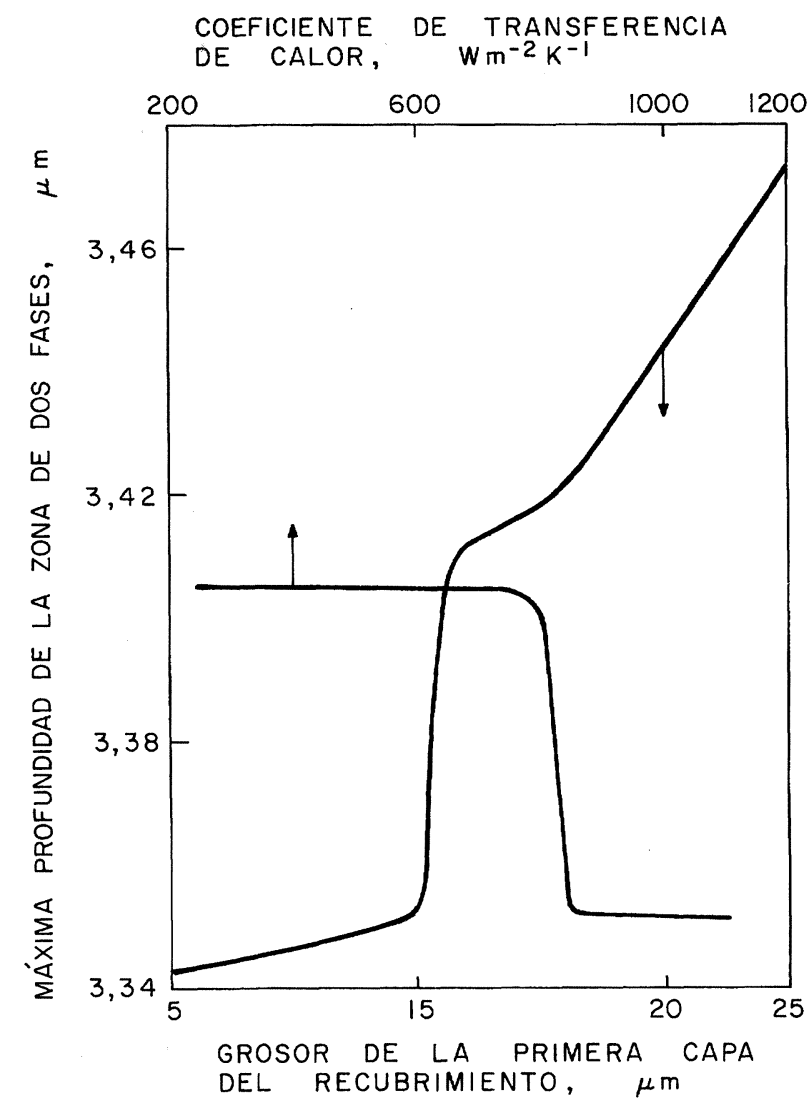

FIG. 9.- Variación de la máxima profundidad de la zona de dos fases en el substrato con respecto al grosor de la primera capa y a $\alpha$.

Fig. 9.- Variation of substrate mushy zone maximal depth with respect to the first layer thickness and $\alpha$.

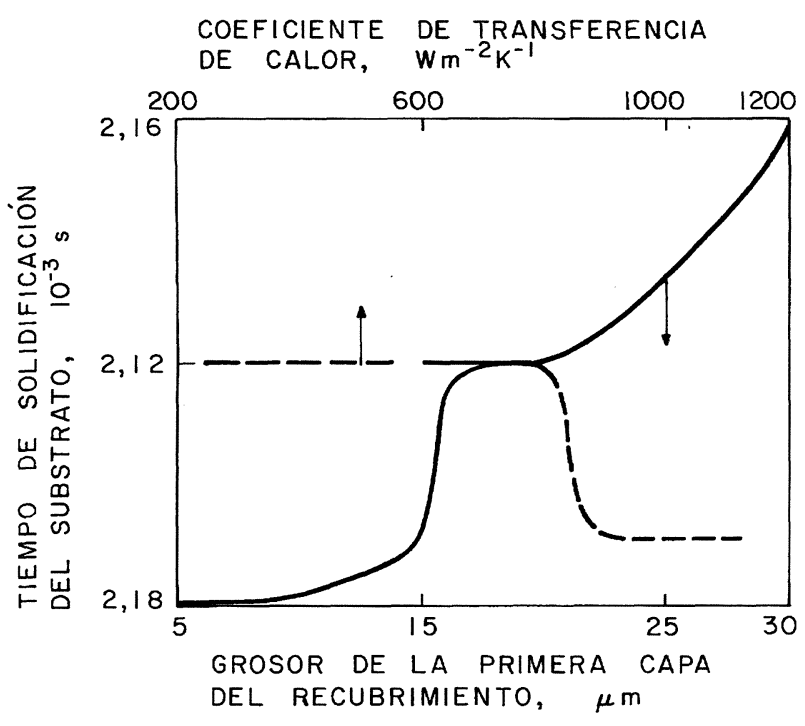

FIG. 10.- Variación del tiempo de solidificación del substrato con respecto al grosor de la primera capa y a $\alpha$.

Fig. 10.- Variation of substrate solidification time with respect to the first layer thickness and $\alpha$.

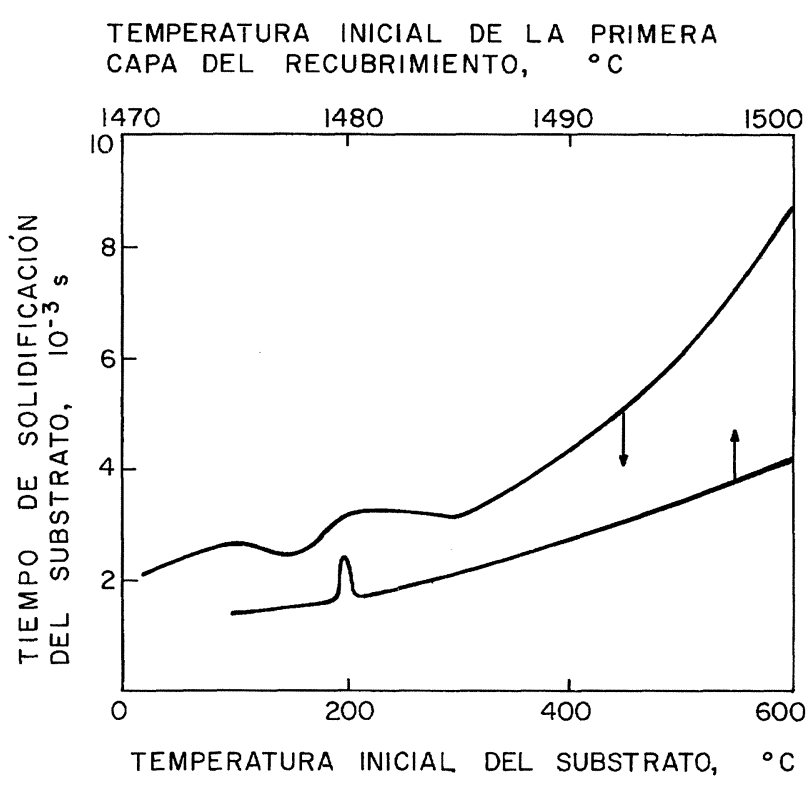

FIG. 11.- Variación del tiempo de solidificación del substrato con respecto a la temperatura inicial del substrato y de la primera capa.

Fig. 11.-Variation of substrate solidification time with respect to the first layer and substrate initial temperatures.

El posterior aumento de $I_{11}$ es resultado de la disminución del crecimiento de la fase sólida en el substrato cuando la situación en la capa permanece casi idéntica. La formación de un plateau cuando $15 \mu \mathrm{m} \leq \delta \leq 17 \mu \mathrm{m}$, está acusadamente relacionada con las características particulares de solidificación de la primera capa de recubrimiento. 


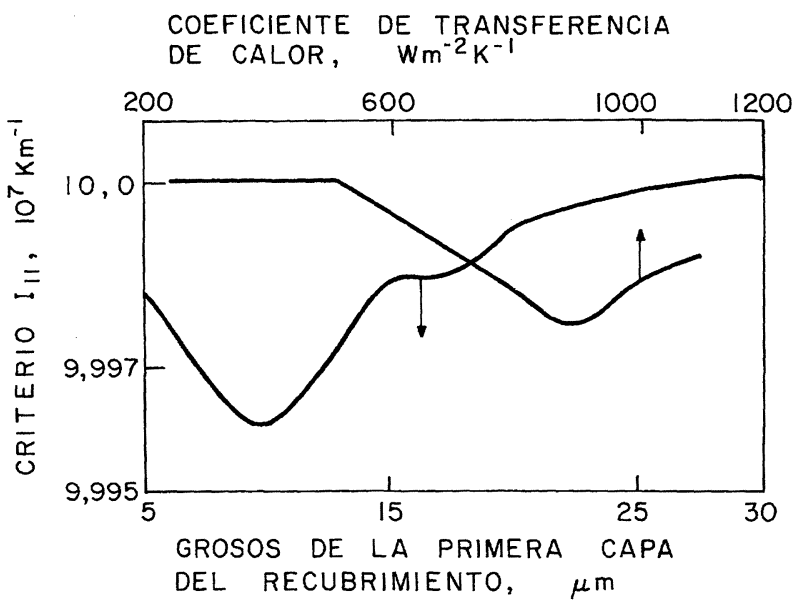

FIG. 12.- Variación del valor del gradiente térmico integral medio en la fase sólida del substrato con respecto al grosor de la primera capa y a $\alpha$.

Fig. 12.-Variation of mean integral value of thermal gradient in substrate solidified solid phase with respect to the first layer thickness and $\alpha$.

La dependencia no uniforme de $I_{11}$ con respecto a $\alpha$, es consecuencia de las variaciones de $t_{\mathrm{s} 1}$ y $t_{\mathrm{s} 2}$ con relación a $\alpha$ (Fig. 4 y 10). El gradiente térmico integral medio en el substrato, $I_{11}$, disminuye con el aumento de la temperatura inicial del substrato y presenta un comportamiento no uniforme respecto a la temperatura inicial del recubrimiento (Fig. 13).

Desde las figuras 12 y 13 se puede observar que las tensiones térmicas mínimas corresponden al valor mínimo de $I_{11}$, lo que debe ocurrir cuando $\delta=10 \mu \mathrm{m}, \alpha=800-1.000 \mathrm{Wm}^{-2} \mathrm{~K}^{-1}, T_{10}=600{ }^{\circ} \mathrm{C}$, $T_{20}=1.490^{\circ} \mathrm{C}$.

El valor del tiempo de residencia integral medio en zona de dos fases, $I_{21}$, varía de forma no uniforme con respecto al grosor de capa $\delta$, alcanzando el valor mínimo durante su evolución (Fig. 14). Esta variación de $I_{21}$ refleja las características mencionadas de la cinética de la solidificación del substrato $\mathrm{y}$, en particular, el máximo de $I_{21}$ se corresponde con el valor mínimo de la velocidad de la isoterma de final de solidificación en el substrato, que determina la máxima región de dos fases. El criterio $I_{21}$ también crece con el aumento de $\alpha$ (Fig. 14).

La figura 15 muestra que el criterio $I_{21}$ disminuye al principio al aumentar la temperatura inicial del substrato, $T_{10}$, alcanzando un mínimo y volviendo a crecer hasta llegar a un nuevo máximo. Este comportamiento de $I_{21}$ se explica con la variación de la mencionada velocidad que se comporta de forma no uniforme con respecto a $T_{10}$. El criterio $I_{21}$ oscila con respecto a $T_{20}$, y su comportamiento es parecido al de $I_{22}$.

Teniendo en cuenta que la posibilidad de formación de defectos estructurales es proporcional a $I_{21}$, se pueden estimar las condiciones óptimas de formación de la estructura del substrato, que corres-
TEMPERATURA INICIAL DE LA PRIMERA

CAPA DEL RECUBRIMIENTO, ${ }^{\circ} \mathrm{C}$

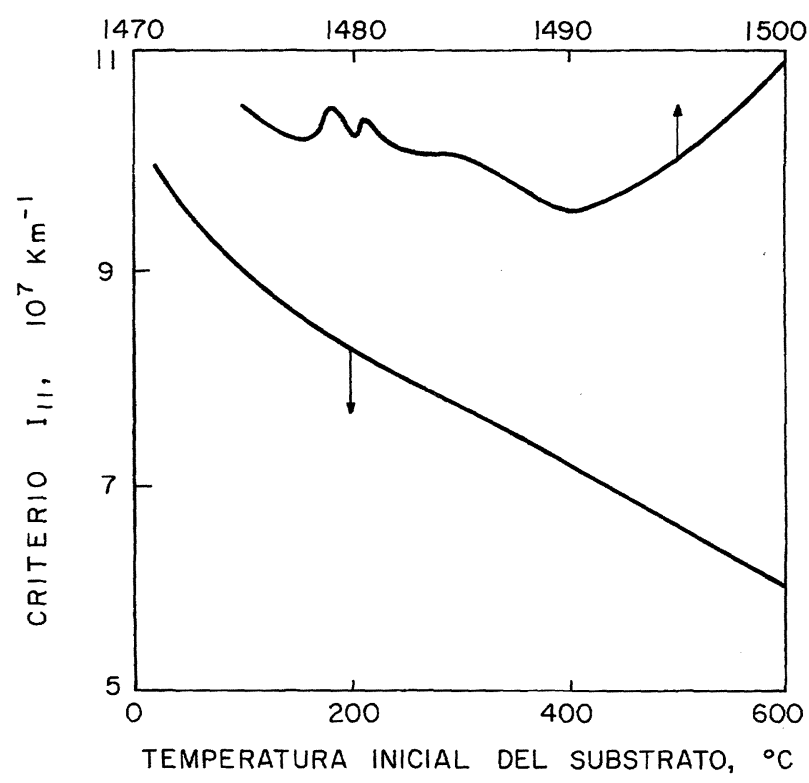

FIG. 13.- Variación del valor del gradiente térmico integral medio en la fase sólida del substrato con respecto a la temperatura inicial del substrato y de la primera capa.

Fig. 13.- Variation of mean integral value of thermal gradient in substrate solidified solid phase with respect to the first layer and substrate initial temperatures.

ponderían a: $\delta=10 \mu \mathrm{m}$ y $19-22 \mu \mathrm{m}, \alpha=300-500$ $\mathrm{Wm}^{-2} \mathrm{~K}^{-1}, T_{10}=100-150{ }^{\circ} \mathrm{C}$ y $400{ }^{\circ} \mathrm{C}, T_{20}=1.481$ ${ }^{\circ} \mathrm{C}$ y $1.490-1.492{ }^{\circ} \mathrm{C}$.

Las condiciones óptimas, desde el punto de vista de formación de tensiones térmicas mínimas y defectos estructurales en el substrato corresponden a: $\delta=10 \mu \mathrm{m}, T_{20}=1.490{ }^{\circ} \mathrm{C}$.

\subsection{Sucesivas capas de recubrimiento}

Después de un tiempo igual al período de rotación del substrato, la segunda capa de recubrimiento llega a la superficie de la primera ya solidificada. Durante el enfriamiento de la segunda capa, esta influye principalmente sobre la primera capa y prácticamente ninguna sobre el substrato. Esto se puede observar en la evolución de la temperatura en la interfase substrato-recubrimiento $(r=R)$.

Durante el período del enfriamiento de la primera capa y antes del depósito de la segunda capa, la temperatura crece en la interfase substrato-recubrimiento debido a la transferencia de calor desde la primera capa, alcanzando su valor máximo cerca de $1.460{ }^{\circ} \mathrm{C}$ relativamente pronto y disminuyendo, posteriormente, como consecuencia de la transferencia de calor hacia todo el volumen del substrato. 


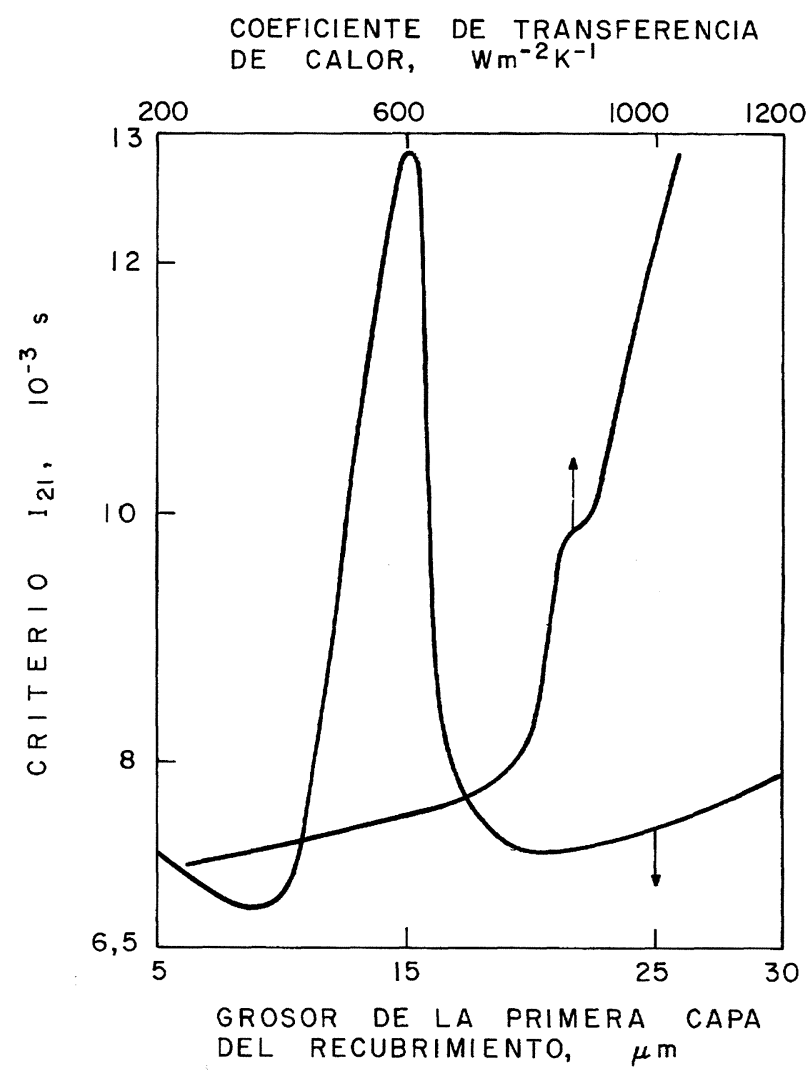

FIG. 14.- Variación del tiempo de residencia medio en zona de dos fases para el substrato con respecto al grosor de la primera capa y a $\alpha$.

Fig. 14.- Variation of mean integral value of residence time in mushy zone of substrate with respect to the first layer thickness and $\alpha$.

En el momento de aparición de la segunda capa, la temperatura de la superficie exterior de la primera capa $\left(r=R_{1}\right)$ es superior a $62{ }^{\circ} \mathrm{C}$. La transferencia de calor desde la segunda capa produce un aumento de la temperatura hasta los $65^{\circ} \mathrm{C}$, y, posteriormente, la intercara entre la primera y la segunda capa se enfría debido a la transferencia de calor hacia el substrato.

Este comportamiento es cualitativamente el mismo que se produce con el depósito de las sucesivas capas. La variación de las temperaturas máximas en estas superficies con respecto al número de capa $m$ muestra que disminuye con $m$, y a partir de $m=3$ no presenta ninguna variación acusada (Fig.16). Esto da a entender que la influencia sobre el sustrato la ofrece principalmente la primera capa de recubrimiento, y las posteriores dinámicas de transferencia de calor en el recubrimiento vienen dadas sólo a través de las interacciones térmicas entre capas vecinas.

\section{CONCLUSIONES}

- El gradiente térmico integral medio en la fase sólida de la primera capa de recubrimiento crece

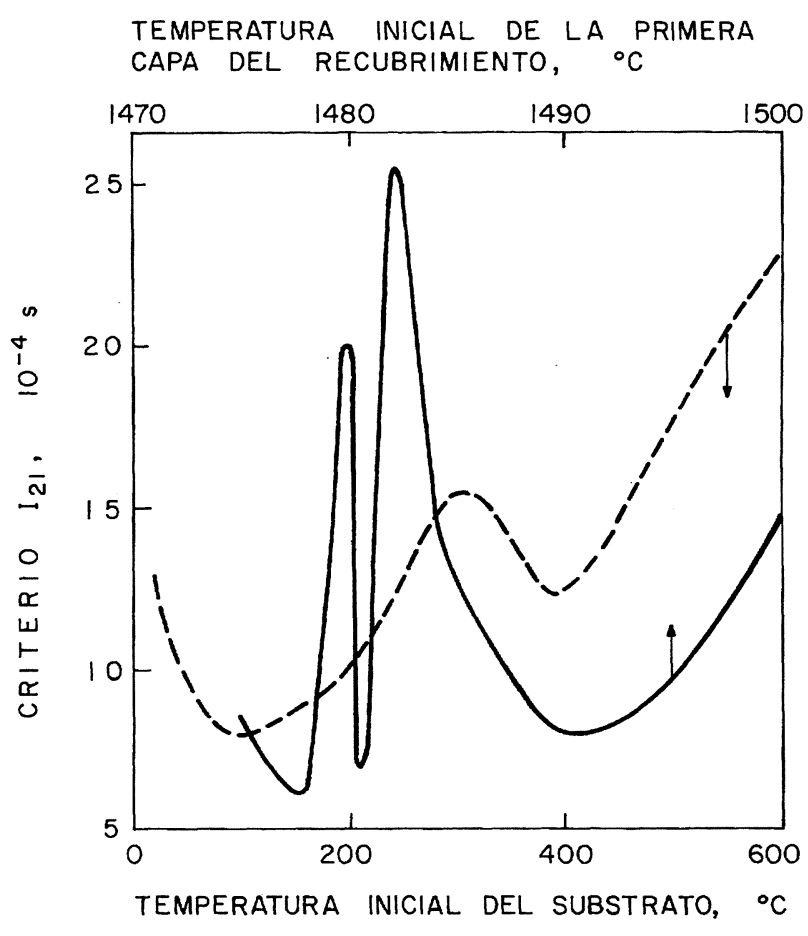

FIG. 15.- Variación del tiempo de residencia medio en zona de dos fases para el substrato con respecto a la temperatura inicial del substrato y de la primera capa.

Fig. 15.- Variation of mean integral value of residence time in mushy zone of substrate with respect to the first layer and substrate initial temperatures.

cuando el grosor de la capa aumenta y $\alpha$ disminuye y sufre una disminución acusada en el intervalo $\alpha=800-850 \mathrm{Wm}^{-2} \mathrm{~K}^{-1}$. Estos gradientes disminuyen con la temperatura inicial del substrato y varían de forma no uniforme con respecto a la temperatura inicial de la primera capa.

- El tiempo de residencia integral medio en zona de dos fases para el recubrimiento muestra un comportamiento no uniforme bajo variaciones del grosor de la capa de recubrimiento, de la temperatura inicial del recubrimiento, así como de la del substrato y de $\alpha$.

- Se puede predecir que las tensiones térmicas mínimas formadas en la primera capa tendrán lugar con, aproximadamente, los siguientes parámetros de proceso: $\delta=5-15 \mu \mathrm{m} ; \alpha=850-1.000 \mathrm{Wm}^{-2} \mathrm{~K}^{-}$ ${ }^{1} ; T_{10}=600^{\circ} \mathrm{C}$ y $T_{20}=1.475^{\circ} \mathrm{C}, 1.500^{\circ} \mathrm{C}$.

- La estructura óptima de capa obtenida durante la solidificación, desde el punto de vista de la aparición de porosidad, inclusiones no metálicas, etc, debería tener lugar cuando $\delta=9-11 \mu \mathrm{m}$, $\alpha=850-1.000 \mathrm{Wm}^{-2} \mathrm{~K}^{-1}, T_{10}=100{ }^{\circ} \mathrm{C}, 550{ }^{\circ} \mathrm{C}$ y $T_{20}=1.480^{\circ} \mathrm{C}$.

- La primera capa de recubrimiento determina en su totalidad la interacción térmica substratorecubrimiento no afectándole de forma 


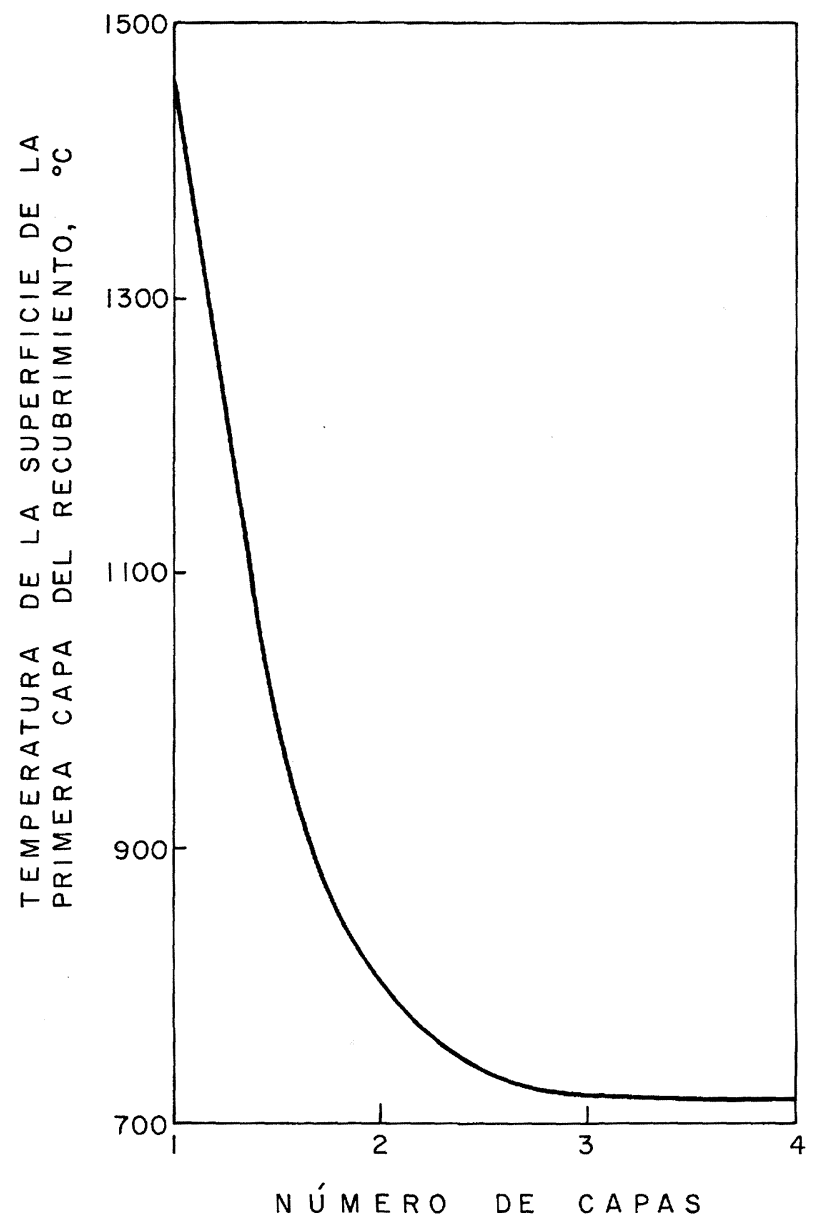

FIG. 16.- Variación de la temperatura de la superficie superior de la primera capa con respecto al número de capas.

Fig. 16.-Variation of temperature at the first layer upper surface with respect to number of layers.

importante las posteriores capas de recubrimiento. Puesto que el substrato resulta influido principalmente por la primera capa de recubrimiento, el calor transferido con posterioridad se determina únicamente a partir de la interacción térmica entre capas vecinas.

- La máxima profundidad de la zona de dos fases disminuye con la transición desde la aleación Co-40 \% W a cobalto puro. Esta profundidad crece con la temperatura inicial del substrato y del recubrimiento, con la disminución de $\alpha$ y con el aumento del grosor de capa de recubrimiento, mostrando, además, un agudo crecimiento en el intervalo de $\delta=15-18 \mu \mathrm{m}$.

- El gradiente térmico integral medio en la fase sólida del substrato disminuye con la temperatura inicial y tiene un comportamiento no uniforme con respecto a $\alpha$, al grosor de capa y a la temperatura inicial del recubrimiento.

- Se puede predecir que las tensiones térmicas mínimas en el substrato se producirán cuando $\delta=10 \mu \mathrm{m}, \alpha=800-1.000 \mathrm{Wm}^{-2} \mathrm{~K}^{-1}, T_{10}=600$

${ }^{\circ} \mathrm{C}, T_{20}=1.490{ }^{\circ} \mathrm{C}$.

- El tiempo de residencia medio en la zona de dos fases para el substrato crece con $\alpha$ y varía no uniformemente con respecto al grosor y temperatura inicial de la capa así como con la temperatura inicial del substrato.

- En la estructura del substrato puede esperarse la parición de los defectos mínimos cuando $\delta=10$ $\mu \mathrm{m}$ y $19-22 \mu \mathrm{m}, \alpha=300-500 \mathrm{Wm}^{-2} \mathrm{~K}^{-1}, T_{10}=$ $100-150{ }^{\circ} \mathrm{C}$ y $400{ }^{\circ} \mathrm{C}, T_{20}=1.481{ }^{\circ} \mathrm{C}$ y $1.490-$ $1.492{ }^{\circ} \mathrm{C}$.

- El desarrollo del modelo matemático permite predecir el estado térmico del substrato y del recubrimiento durante la proyección HVOF así como las condiciones óptimas de formación de su estructura, lo cual permite ajustar los parámetros de proyección para minimizar los defectos estructurales más importantes.

\section{Agradecimiento}

Los autores agradecen a la Generalitat de Catalunya y a Carburos Metálicos, S.A., el soporte financiero recibido con el proyecto GRQ93-1017. V.V. Sobolev agradece también a la CICYT la concesión del Año Sabático SAB 94-0066 en la Universidad de Barcelona. J. A. Calero expresa su gratitud a la Generalitat de Catalunya por la concesión de una beca de F.I.

\section{REFERENCIAS}

(1) Parker, D.W. y Kutner, G.L. Adv. Mater. Proces., 7, 1994: 31-35.

(2) THORPE, M.L. y RichteR, H.J.J. Therm. Spray Technol., 1 (2), 1992: 161-170.

(3) Sobolev, V.V., Guilemany, J.M. y Calero, J.A. Surf. Coat. Technol., 63, 1994: 181-187.

(4) Sobolev, V.V., Guilemany, J.M. y Calero, J.A. J. Therm. Spray Technol, 1994. En prensa.

(5) Sobolev, V.V. y Guilemany, J.M. Mater. Lett., 18, 1994: 304-308.

(6) Sobolev, V.V. y Guilemany, J.M. Mater. Proces. Technol. 1994. En prensa.

(7) Sobolev, V.V. y Guilemany, J.M. Surf. Coat. Technol., 70, 1994: 57-68.

(8) Apelian, D., Paliwal, M., Smith, R.W. y Schilling, W.F. Inter. Met. Rev., 28 (5), 1994: 271-294.

(9) Cirolini, S., Harding, J.H. y Jacucci, G. Surf. Coat. Technol., 48, 1991: 137-145.

(10) Moreau, C., Lamontagne, M. y Cielo, P. Surf. Coat. Technol., 53, 1992: 107-114.

(11) Sobolev, V.V. y TrefILOV, P.M. Thermophysics of metal solidification during continuous casting. Ed. Metallurgy Publ. Co. Moscú, 1988.

(12) Sobolev, V.V. y Trefilov, P.M. Optimization of thermal conditions of melts solidification. Ed. Krasnoyarsk University Press. Krasnoyarsk (CEI), 1986.

(13) Metals Handbook. 10 ed. ASM International. NuevaYork, 1990.

(14) Chapman, A.J. Heat transfer. Macmillan Publ. Co. Nueva-York, 1984. 